\title{
ANÁLISE POSTURAL DE INDIVÍDUOS PORTADORES DE DEFICIÊNCIA VISUAL E AUDITIVA
}

José Carlos de LIMA JÚNIOR ${ }^{1}$

Vivaldo Xavier Silva SOUSA ${ }^{2}$

\begin{abstract}
${ }^{1}$ Fisioterapeuta graduado pelo Centro de Ensino Unificado de Teresina - CEUT, Pós Graduando em Terapia Intensiva pelo Hospital São Paulo/Instituto Camilo Filho - HSP/ICF, em Teresina, PI - Brasil. Email: lima_juniorviana@ hotmail.com
\end{abstract}

${ }^{2}$ Fisioterapeuta graduado pela Associação de Ensino Superior do Piauí - AESPI, Mestre em Engenharia Biomédica pela Universidade do Vale do Paraíba - UNIVAP, São José dos Campos, SP - Brasil. Email: fisioxavier@ hotmail.com

Recebido em: 08/01/2015 - Aprovado em: 15/06/2015 - Disponibilizado em: 15/07/2015

\begin{abstract}
RESUMO
Introdução: A visão e a audição são os maiores responsáveis por receber informações do ambiente, informações essas necessárias para a manutenção do controle postural. Desta forma, pressupõe-se que indivíduos com deficiência visual e/ou auditiva tornam-se propensos a apresentar controle postural prejudicado, podendo desenvolver estratégias posturais diferentes daqueles sem deficiência. Objetivo: Analisar a postural de indivíduos portadores de deficiência visual e auditiva. Metodologia: Participaram do estudo quarenta e cinco indivíduos, entre 18 e 45 anos, distribuídos em três grupos: Grupo com Deficiência Visual (GDV) formado por 15 deficientes visuais, Grupo com Deficiência Auditiva (GDA) formado por 15 deficientes auditivos e Grupo com Indivíduos Normais (GIN), grupo controle, formado por 15 pessoas de visão e audição normais. A avaliação postural foi efetuada com o software de avaliação postural (SAPO). Resultados: Foram encontradas diferenças significativas no alinhamento horizontal dos acrômios, entre o GDV e GIN. A análise demonstrou também diferença significativa no alinhamento vertical da cabeça onde o GDV apresentou maior desvio. Conclusão: $\mathrm{O}$ estudo revela assimetrias estáticas em pessoas com deficiência visual, quando comparadas com pessoas com deficiência auditiva e indivíduos normais.
\end{abstract}

Palavras-chave: Postura corporal. Baixa visão. Perda auditiva.Pessoas com deficiência visual.Pessoas com deficiênciaauditiva.

\begin{abstract}
Introduction: The vision and hearing are the most responsible for receiving information from the environment, such information necessary for the maintenance of postural control. Thus, it is assumed that individuals with visual and / or hearing impairment become prone to impaired postural control and may develop different postural strategies of those without disabilities.Objective: To analyze the posture of individuals with visual and hearing impairment. Methodology: The study included forty-five individuals, between 18 and 45 years, divided into three groups: Group with Visual Impairment (GVI) consisting of 15 visually impaired Group for Hearing Impaired (GHI) formed by 15 deaf and Group Individuals with Standard (GIS), control group was formed by 15 people of vision and normal hearing. Postural assessment was performed for the postural assessment software (SAPO).Results: Significant differences were found in the horizontal alignment of acromions between the GDV and GIN. The analysis also showed a significant difference in the vertical alignment of the head where the GDV showed greater deviation. Conclusion: The study reveals static asymmetry in people with visual impairment, compared with people with hearing impairment and normal subjects.
\end{abstract}

Keywords: Body posture. Low vision.Hearing loss.People with visual impairments.People withhearingdisabilities.

\section{INTRODUÇÃO}

De acordo com dados do IBGE, obtidos no Censo Demográfico realizado em 2010, no Brasil mais 45,6 milhões de pessoas apresentam algum tipo de deficiência. A deficiência visual é a mais frequente, onde aproximadamente 6,5 milhões possuem dificuldade severa, 6 milhões dificuldade de enxergar e 506 mil cegos. Em relação à deficiência auditivaos números constatados foram de cerca de 9,7 milhões, sendo 2,1 milhões de pessoas apresentam de forma severa, 1,7 milhão possuem grande dificuldade 
de ouvir e 344,2 mil são surdas(INSTITUTO

BRASILEIRO DE GEOGRAFIA E ESTATÍSTICA, 2014).

A visão e a audição são os maiores responsáveis por receber informações do ambiente (SANCHEZet al., 2008), informações essas necessárias para a manutenção do controle postural. Juntas desempenham um importante papel na estabilização da postura, devidofornecer continuamente ao sistema nervoso central informações a respeito da direção e velocidade dos movimentos e da posição dos segmentos do corpo em relação a eles mesmos e ao ambiente (EASTONet al., 1998; BRICOT, 2011; SILVA et al., 2011).

A postura é um complexo mecanismo sensório-motor e base para todo movimento (LOTH et al., 2008).Três sistemas auxiliam na manutenção da postura: o visual, o vestibular, e o somatossensorial (MERGNER et al., 2005). Se um desses sistemas falha, segundo Nakata e Yabe (2001), a postura fica dificultada e aumenta a oscilação corporal.

Desta forma, pressupõe-se que indivíduos com deficiência visual e/ou auditiva tornam-se propensos a apresentar controle postural prejudicado (SILVA et al., 2011), podendo desenvolver estratégias posturais diferentes daqueles sem deficiência. Essas estratégias, embora tenham afinalidade de obter uma maior estabilidade postural, podem favorecer o desenvolvimento de alterações posturais(CASCIO, 2010;
SOUSAet al., 2010; SCHWESIG et al., 2011).

Apesar do grande número de portadores de deficiência visual e auditiva no Brasil e no mundo, ainda sabe-se pouco sobre quais as consequências de uma informação visual e auditiva ausente ou deficiente para a manutenção da postura nesta população (MASCARENHASet al., 2009; MELO et al., 2011).Neste contexto, o objetivo do estudo foi analisar a postural de indivíduos portadores de deficiência visual e auditiva.

\section{METODOLOGIA}

Trata-se de uma pesquisa observacional transversal quantitativa. Participaram da pesquisa quarenta e cinco voluntários, de faixa etária de 18 e 45 anos, de ambos os gêneros. Eles foram distribuídos em três grupos: Grupo com Deficiência Visual (GDV) formado por 15deficientes visuais, Grupo com Deficiência Auditiva (GDA) formado por 15 deficientes auditivos e Grupo com Indivíduos Normais (GIN), grupo controle, formado por 15 pessoas de visão e audição normais.

Os critérios de inclusão para o grupo formado por deficientes visuais foram: indivíduos com deficiência visual (cegas), sedentários e que não apresentaram comprometimento do sistema vestibular, proprioceptivo, auditivo, neurológico e/ou mental. 
Para o grupo formado por deficientes auditivosos critérios de inclusão foram: indivíduos com deficiência auditiva (surdez), sedentários e que não apresentaram comprometimento do sistema vestibular, proprioceptivo, auditivo, neurológico e/ou mental.

Já para o grupo formado por pessoas com visão normal os critérios de inclusão foram: indivíduos com visão e audição normal, sedentários e que não apresentaram comprometimento do sistema vestibular, proprioceptivo, auditivo, neurológico e/ou mental.

Foram adotados como critérios de exclusão, paraos três grupos, as características listadas a seguir: indivíduos com presença de comprometimento físico, neurológico e/ou mental, portadores de labirintite, praticantes de atividade física, mulheres grávidas e ausência de colaboração nos procedimentos a serem realizados.

A pesquisa foi realizada obedecendo às normas éticas da Resolução 196/96 do Conselho Nacional de Saúde, que dispõe sobre pesquisa realizada com seres humanos e foi aprovada pelo Comitê de Ética em Pesquisa local (protocolo $\mathrm{n}^{\circ}$ 182/2013). Antes do início do estudo, todos os participantes foram informados dos procedimentos de avaliação necessários e consentiram em participar de forma voluntária por meio de assinatura do Termo de Consentimento Livre e Esclarecido (TCLE).
Inicialmente foi realizada uma rápida anamnese onde foi coletado: nome, idade, medidas antropométricase IMC de cada voluntário. As medidas antropométricas foram avaliadas a partir da medida da massa corporal e estatura por meio da balança digitalFilizola $^{\circledR}$, com estadiômetro acoplado, e o IMC foi calculado dividindo-se o peso pela altura ao quadrado.Em seguida foi utilizado a analise postural através do Software de Avaliação Postural (SAPO $\left.{ }^{\circledR}\right)$ versão 0,68.

O SAPO ${ }^{\circledR}$ avalia a postura por meio da fotogrametria, que a partir de fotos possibilita medidas de posição, comprimento, ângulo, centro de gravidade e alinhamento corporal. Possui fácil aplicação e é disponibilizadode forma gratuita na internet. O seu protocolo é uma sugestão de pontos de marcação e medidas para avaliação postural, baseada na relevância clínica, base científica, viabilidade metodológica e aplicabilidade (GLANERet al., 2012).

Os pontos anatômicos prédeterminados no protocolo $\mathrm{SAPO}^{\circledR}$ foram demarcados com bolas brancas de isopor de $15 \mathrm{~mm}$ de diâmetro, fixadas nos voluntários com fita dupla face. Os pontos demarcados (bilateralmente) foram: lóbulos da orelha, acrômio, espinha ilíaca ântero-superior, trocanter maior do fêmur, linha articular do joelho, borda superior da patela, tuberosidade da tíbia, maléolo medial e lateral, ponto entre a cabeça do segundo e terceiro metatarso, borda inferior da escápula, espinha ilíaca 
póstero-superior, calcâneo, tendão do calcâneo, linha média posterior da tíbia, processos espinhosos da $7^{\mathrm{a}}$ vértebra cervical (C7) e da $3^{\text {a }}$ vértebra torácica (T3).

A partir dos pontos demarcados foram adquiridas as seguintes medidas: alinhamento horizontal da cabeça (AHCA), alinhamento horizontal dos acrômios (AHA), alinhamento horizontal das espinhas ilíacas ânterosuperiores (AHEIAS), ângulo dos dois acrômios e das duas espinhas ilíacas ânterosuperiores (ADAEIAS), ângulo frontal do membro inferior direito e esquerdo (AFMID e AFMIE), diferença de comprimento nos membros inferiores (DCMI), alinhamento horizontal das tuberosidades das tíbias (AHTT), ângulo $\mathrm{Q}$ direito (AQD) e ângulo $\mathrm{Q}$ esquerdo (AQE), assimetria horizontal da escápula em relação a T3 (AHET3), ângulo perna/retropé direito (APRD) e ângulo perna/retropé esquerdo (APRE), alinhamento horizontal da cabeça em relação à C7 lado direito e esquerdo (AHCLD e AHCLE), alinhamento vertical da cabeça em relação ao acrômio lado direito e esquerdo (AVCLD e AVCLE), alinhamento vertical do tronco lado direito e esquerdo (AVTLD e AVTLE), ângulo do quadril (tronco e membro inferior) lado direito e esquerdo (AQLD e AQLE), alinhamento vertical do corpo lado direito e esquerdo (AVCOLD e AVCOLE), alinhamento horizontal da pélvis lado direito e esquerdo (AHPLD e AHPLE), ângulo do joelho lado direito e esquerdo (AJLD e
AJLE), ângulo do tornozelo lado direito e esquerdo (ATLD e ATLE).

Para a realização dos registos fotográficos os voluntários permaneceram em posição ortostática ao lado de um fio de prumo, posicionadas no local marcado previamente com um tapete em etil vinil acetato de $70 \times 74 \mathrm{~cm}$, no qual foi feito com giz branco o contorno dos pés de cada voluntária, para assegurar o mesmo posicionamento nas diferentes vistas de coleta. As imagens foram capturadas com uma câmera fotográfica digital da marca Sony modelo DSC-W220 de 12.1 mega pixels posicionada paralela ao chão, sobre um tripé nivelado e a $3 \mathrm{~m}$ dos avaliados. A altura da câmera foi fixada na metade da estatura de cada voluntário. A sala possuía fundo preto e apresentava boa iluminação, como sugereo protocolo.

Foram realizadas quatro fotos de cada indivíduo: 1 em vista anterior, 1 em vista lateral direita, 1 em vista lateral esquerda e 1 em vista posterior. Por meio delas e com o auxilio do $\mathrm{SAPO}^{\circledR}$, foram feitas as análises posturais das 45 pessoas.

$\mathrm{Na}$ analise estatística foi utilizado o programa estatístico SPSS 15.0 para Windows. Foram realizadosanálises descritivas e o teste de normalidade. Para verificar as possíveis diferenças entre os grupos em relação às variáveis posturais foi utilizado o teste não paramétrico $t$ de Student independente. O nível de significância 
adotado foi $\mathrm{p}<0,05$. Todos os dados foram expressos em média e Desvio Padrão (DP).

\section{RESULTADOS}

Não houve diferença estatisticamente significativa entre as idades, às medidas antropométricas e o IMC dos participantes avaliados nesta pesquisa.

As comparações entre os grupos para as variáveis da postura nas diferentes vistas estão expressos nas tabelas a seguir.

Tabela 1 -Comparação da vista anterior entre os grupos.

\begin{tabular}{lcccc}
\hline \multirow{1}{*}{ Variável Estudada } & GDV & GDA & GVN & \\
\cline { 2 - 4 } & M \pm DP & M \pm DP & M \pm DP & \\
\hline Alinhamento horizontal da cabeça & $4,8 \pm 3,7$ & $3,5 \pm 1,6$ & $2,3 \pm 1,4$ & 0,0600 \\
Alinhamento horizontal dos acrômios & $3,1 \pm 1,4$ & $2,1 \pm 1,3$ & $1,3 \pm 0,6$ & $0,0200^{*}$ \\
Alinhamento horizontal das EIAS & $1,7 \pm 1,4$ & $1,9 \pm 1,1$ & $1,5 \pm 0,9$ & 0,5600 \\
Diferença de comprimento MMII (cm) & $1,1 \pm 0,7$ & $1,5 \pm 1,2$ & $0,9 \pm 0,7$ & 0,0500 \\
Ângulo Q direito & $11,1 \pm 8,5$ & $12,4 \pm 8,2$ & $16,6 \pm 6,5$ & 0,1200 \\
Ângulo Q esquerdo & $15,0 \pm 9,2$ & $12,8 \pm 7,7$ & $12,0 \pm 6,7$ & 0,2400
\end{tabular}

Legenda: GDV - Grupo com Deficiência Visual, GDA - Grupo com Deficiência Auditiva, GVN - Grupo com Visão Normal, M \pm DP- média e Desvio Padrão, * Diferença significativa entre os grupos.Fonte: Dados de produção do próprio autor.

A análise da vista anterior demonstrou diferença significativa no alinhamento horizontal dos acrômios $(\mathrm{p}=0,0200)$ onde o GDV apresentou maior desvio que o GVN.

Tabela 2 - Comparação da vista lateral direita entre os grupos.

\begin{tabular}{lcccc}
\hline \multirow{2}{*}{ Variável Estudada } & GDV & GDA & GVN & \multirow{2}{*}{$\boldsymbol{p}$} \\
\cline { 2 - 4 } & $\mathbf{M} \pm \mathbf{D P}$ & $\mathbf{M} \pm \mathbf{D P}$ & $\mathbf{M} \pm \mathbf{D P}$ & \\
\hline Alinhamento vertical da cabeça & $20,8 \pm 11,6$ & $12,5 \pm 9,5$ & $10,5 \pm 8,3$ & $0,0300^{*}$ \\
Alinhamento vertical do tronco & $2,3 \pm 1,4$ & $2,2 \pm 1,3$ & $1,9 \pm 0,7$ & 0,1800 \\
Alinhamento horizontal da pélvis & $10,7 \pm 8,8$ & $8,1 \pm 3,6$ & $5,8 \pm 3,9$ & 0,1700 \\
GenuFlexo/Recurvatum & $4,0 \pm 3,0$ & $3,1 \pm 2,9$ & $2,6 \pm 2,3$ & 0,0800 \\
\hline
\end{tabular}

Legenda: GDV - Grupo com Deficiência Visual, GDA - Grupo com Deficiência Auditiva, GVN - Grupo com Visão Normal, M DPP - média e Desvio Padrão, * Diferença significativa entre os grupos.Fonte: Dados de produção do próprio autor. 
A análise da vista lateral direita os GDA e GVN apresentaram menor desvio demonstrou diferença significativa no que o GDV.

alinhamento vertical da cabeça $(\mathrm{p}=0,03)$ onde

Tabela 3 - Comparação da vista lateral esquerda entre os grupos.

\begin{tabular}{lcccc}
\hline \multirow{2}{*}{ Variável Estudada } & GDV & GDA & GIN & \multirow{2}{*}{$\boldsymbol{p}$} \\
\cline { 2 - 4 } & $\mathbf{M} \pm \mathbf{D P}$ & $\mathbf{M} \pm \mathbf{D P}$ & $\mathbf{M} \pm \mathbf{D P}$ & \\
\hline Alinhamento vertical da cabeça & $12,4 \pm 14,3$ & $17,9 \pm 11,7$ & $16,9 \pm 12,7$ & 0,0900 \\
Alinhamento vertical do tronco & $3,2 \pm 1,5$ & $2,5 \pm 1,4$ & $1,8 \pm 0,9$ & 0,1400 \\
Alinhamento horizontal da pélvis & $12,9 \pm 6,8$ & $8,1 \pm 5,6$ & $6,7 \pm 4,8$ & 0,3900 \\
GenuFlexo/Recurvatum & $3,6 \pm 2,8$ & $3,3 \pm 2,8$ & $3,0 \pm 2,4$ & 0,8300 \\
\hline
\end{tabular}

Legenda: GDV - Grupo com Deficiência Visual, GDA - Grupo com Deficiência Auditiva, GVN - Grupo com Visão Normal, M \pm DP - média e Desvio Padrão.Fonte: Dados de produção do próprio autor.

Tabela 4 - Comparação da vista posterior entre os grupos.

\begin{tabular}{lcccc}
\hline \multirow{2}{*}{ Variável Estudada } & GDV & GDA & GIN & \multirow{2}{*}{$\boldsymbol{p}$} \\
\cline { 2 - 4 } & $\mathbf{M} \pm \mathbf{D P}$ & $\mathbf{M} \pm \mathbf{D P}$ & $\mathbf{M} \pm \mathbf{D P}$ & \\
\hline Valgo/Varo Calcâneo Direito & $7,1 \pm 5,6$ & $5,8 \pm 4,3$ & $5,4 \pm 4,1$ & 0,7400 \\
Valgo/Varo Calcâneo Esquerdo & $10,0, \pm 9,7$ & $9,2 \pm 4,7$ & $5,9 \pm 4,7$ & 0,4100
\end{tabular}

Legenda: GDV - Grupo com Deficiência Visual, GDA - Grupo com Deficiência Auditiva, GVN - Grupo com Visão Normal, M DDP - média e Desvio Padrão.Fonte: Dados de produção do próprio autor.

Para as demais comparações, não houve diferença estatística entre os grupos $(\mathrm{p} \geq 0,05)$.

\section{DISCUSSÃO}

A partir da investigação realizada neste estudo, sobre a presença e os tipos de alterações posturais em indivíduos com deficiência visual e auditiva, percebeu-se que essas alterações são muito prevalentes na população de deficientes visuais, como relaram outros autores (MERGNERet al., 2005; SANCHEZ et al., 2008; SILVA et al.,
2011).Contudo, apesar da não identificação de diferença estatisticamente significante na avaliação da postura dos voluntários com deficiência auditiva e de não haver estudos nesta população com a fotogrametria, existem estudos na literatura afirmam que há presença dealterações posturais nestes indivíduos (MASCARENHAS et al., 2009; MELO et al., 2011).

Detalhando os resultados obtidos, observou-se na análise das variáveis estudadas, diferença no alinhamento horizontal dos acrômios (assimetria dos ombros), onde se notou diferença significativa 
entre os grupos GAV e GVN, com valores maiores para o primeiro, além da diferença no alinhamento vertical da cabeça, onde os GDA e GVNapresentaram menor desvio que o GDV, na comparação realizada entre eles na vista lateral direita.

Foram encontrados na literatura estudos que concordam com esses achados. Amaya e De Cabrera (1991)e Prechtlet al. (2001)identificaram algumas alterações comuns a indivíduos com deficiência visual, entre elas as alterações na coluna vertebral e assimetrias de ombro. Esses autores concluíram que a presença dessa alteração postural ocorre em consequência do comprometimento visual.

O estudo de Sanchez et al. (2008)também apresentou alterações de simetria nos ombros. Relataram que isso pode ser resultado da hipertrofia de determinados grupos musculares sobre outros. Esta hipertrofia pode induzir a alterações posturais devido à tensão da musculatura das costas e a necessidade de se manter alerta para compensar a falta de visão.

Em relação à diferença no alinhamento vertical da cabeça mostrado neste estudo, Arcanjo et al. (2005)afirma que um déficit visual contribui para o surgimento de alterações posturais e inclui nestas as inclinações de cabeça, tronco e cintura pélvica. Gerente, Pascoal e Pereira (2008)constataram em seu estudo com cegos e videntes, que os indivíduos cegos realizam uma rotação e inclinação da cabeça a fim de localizar estímulo sonoro posicionado frontalmente.

Simprini e Braccialli (1998)realizaram uma pesquisa sobre a influência do sistema sensório-motor na manutenção da postura estática em indivíduos cegos e observaram haver a presença de rotação e inclinação da cabeça para o lado direito e alteração na consciência corporal.Também mostraram diferença quanto à presença de inclinação assimetria de ombros. Esses autores sugerem que alteração na posição da cabeça poderia ser decorrente dos ajustes feitos para que se atingir a melhor percepção auditiva.

Em uma pesquisa realizada por Silva et al. (2011)para avaliar a ocorrência de alterações posturais e retrações musculares em um portador de deficiência visual parcial, foi percebido que os portadores de deficiência visual apresentam um aumento nas assimetrias posturais e principalmente na curvatura escoliótica e isso é o resultado de uma postura anormal de cabeça, em que o objetivo é melhorar o ângulo da visão. As alterações posturais mais evidentes como rotação de tronco e desalinhamento da cabeça, evidenciadas pela inclinação com rotação da coluna cervical eanteriorizaçãode cabeça podem ser consequentes da ausência de referência horizontal característica dos deficientes visuais. Nessa condição não há a necessidade de adequação postural pelo indivíduo para correção visual, havendo uma 
alteração postural mais nítida do que normalmente é encontrada nas avaliações posturais de indivíduos videntes (SHABANA et al., 2005; FRIEDRICH et al., 2008).

Diante dos achados, a visão mostrouse ser mais determinante que a audição para a regulação da postura corporal, já que não houve alterações no grupo dos deficientes auditivos, quando comparado com o próprio grupo de deficientes visuais e com o grupo dos indivíduos normais.Logo, a visão identificou-se como sendo um sistema sensorial primordial e indispensável para a manutenção da postura.

\section{CONCLUSÃO}

Mediante os resultados encontrados, observou-se a presença de alterações posturais em indivíduos cegos quando comparados aos indivíduos surdos e pessoas normais, demonstrando que a visão é, portanto, fator fundamental no ajuste postural.Foi verificado que indivíduos com cegueira apresentam assimetria no alinhamento horizontal dos acrômios quando comparados a pessoas normais e alteração no alinhamento vertical da cabeça, quando comparado a pessoas com deficiência auditiva e indivíduos normais.

Reconhece-se que o tamanho da população investigada pode ter sido um fator limitante deste estudo, assim, os nossos achados não podem representar dados conclusivos. Por outro lado, a insuficiência de pesquisas publicadasobre a análiseda postura de indivíduos portadores de deficiência visual e auditiva reforça a necessidade de novos estudos.

\section{REFERÊNCIAS}

ARCANJO, G. N. et al. Alterações da relação entre mudanças posturais na coluna e alterações visuais. Fisioter. Bras., Rio de Janeiro, v. 6, n. 5, p. 354-360, set./out. 2005.

AMAYA, J. M. M.; DE CABRERA, B. C. Postura em niños videntes e invidentes congênitos. Acta Pediátr. Méx.,Cidade do México, v. 12, n. 3, p. 136-147, may./jun. 1991.

BRICOT, B. Posturologiaclínica. São Paulo: CIES Brasil, 2011.

CASCIO, C. J. Somatosensory processing in neurodevelopmental disorders.

J.Neurodev.Disord., North Carolina,v. 2, n. 2, p. 62-69, jun. 2010.

EASTON, R. D. et al. Auditory cues for orientation and postural control in sighted and congenitally blind people. Exp. Brain. Res., Berlin, v.118, n. 4, p. 541-550, feb. 1998.

FRIEDRICH, M. et al. Influence of pathologic and simulated visual dysfunctions 
on the postural system. Exp Brain Res.,

Berlin, v. 186, n. 2, p. 305-314, mar. 2008.

GERENTE, J. G. S.; PASCOAL, A. G.;

PEREIRA, M. L. M. Localização espacial de

estímulos sonoros em indivíduos cegos

congênitos: estudo comparativo da posição

tridimensional da cabeça em adultos cegos

congênitos e indivíduos videntes. Rev. Bras.

Educ. Espec., Piracicaba, v. 14, n. 1, p. 111-

120, jan./abr. 2008.

GLANER, M. F. et al. Fotogrametria:

fidedignidade e falta de objetividade na

avaliação postural. Motricidade.,Santa Maria

da Feira, v. 8, n. 1, p. 78-85, 2012.

\section{INSTITUTO BRASILEIRO DE}

GEOGRAFIA E ESTATÍSTICA. População:

Censo Demográfico 2010. Acesso em: 12

dez 2014. Disponível em:

<http://www.ibge.gov.br/home/mapa_site/ma

pa_site.php\#populacao >.

LOTH, E. D. et al. Avaliação da influência do sistema vestibular no equilíbrio de adultos jovens através da posturografia dinâmica foam-laser e plataforma de força. Sem:

CiencBiologSaud., Londrina, v. 29, n. 1, p. 57-68, jan./jun. 2008.

MASCARENHAS, C. H. M. et al. Alterações posturais em deficientes visuais no município de Jequié/BA. Rev. Espaço para a Saúde, Londrina, v. 11, n. 1, p. 1-7, dez. 2009.

MELO, R. S. et al.Avaliação postural da coluna vertebral em crianças e adolescentes com deficiência auditiva. Arq.

Int.Otorrinolaringol., São Paulo, v. 15, n. 2, p. 195-202, abr./jun. 2011.

MERGNER, T. et al. Human postural responses to motion of real and virtual visual environments under different support base conditions.Exp. Brain.Res., Berlin, v. 167, n. 4, p. 535, 556, dec. 2005.

NAKATA, H.; KYONOSUKE, Y. B. Automatic postural response systems in individuals with congenital total blindness.Gait Posture.,Oxford, v. 14, n. 1, p. 36-43, jul. 2001.

PRECHTL, H. F. R. et al. Role of vision on early motor development: lessons from the blind. Dev. Med.Child.Neurol., London, v. 43, n. 3, p. 198-201, 2001.

SANCHEZ, H. M.et al. Avaliação postural de indivíduos portadores de deficiência visual através da biofotogrametria computadorizada. Fisioter.Mov.,Rio de Janeiro, v. 21, n. 2, p. 11-20, abr./jun. 2008.

SCHWESIG, R. et al. Postural control in subjects with visual impairment.Eur. 
J.Ophthalmol.,Milano, v. 21, n. 3, p. 303-

309, may./jun. 2011.

SILVA, M. B. et al.Avaliação das alterações

posturais e retrações musculares na

deficiência visual: estudo de caso. Saúde

Coletiva, São Paulo, v. 49, n. 8, p. 77-82,

2011.

SIMPRINI, R.; BRACCIALLI, L. P.

Influência do sistema sensório-motor na

manutenção da postura estática em indivíduos

cegos. Rev.Neuropsiq.da Inf. e Adol., v. 6,

n. 1, p. 26-38, 1998.

SHABANA, N. et al.Postural stability in

primary open angle

glaucoma.Clin.Experiment.Ophthalmol.,

Carlton, v. 33, n. 3, p. 264-273, jun. 2005.

SOUSA, A. M. M. et al. Avaliação do

controle postural e do equilíbrio em crianças

com deficiência auditiva. R. da EducFis

/UEM., Maringá, v. 21, n. 1, p. 47-57, 2010. 